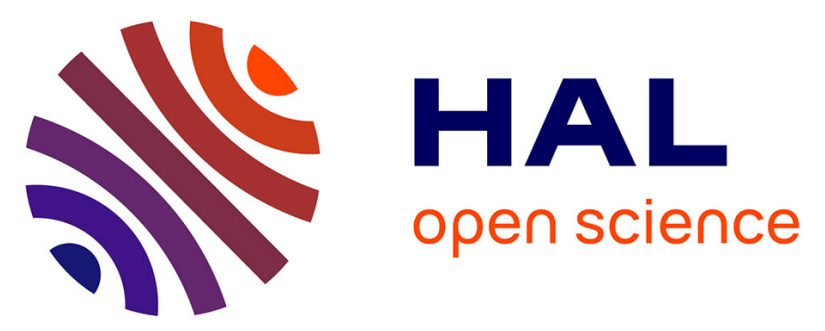

\title{
Relations parcours-énergie des ions lourds de faible vitesse dans les détecteurs visuels plastiques. Étude particulière des fragments de fission
}

J. Ralarosy, R. Schmitt, G. Mosinski, J. Tripier, M. Debeauvais, R. Stein, G. Rémy

\section{To cite this version:}

J. Ralarosy, R. Schmitt, G. Mosinski, J. Tripier, M. Debeauvais, et al.. Relations parcours-énergie des ions lourds de faible vitesse dans les détecteurs visuels plastiques. Étude particulière des fragments de fission. Journal de Physique, 1969, 30 (1), pp.1-13. 10.1051/jphys:019690030010100 . jpa-00206759

\section{HAL Id: jpa-00206759 https://hal.science/jpa-00206759}

Submitted on 1 Jan 1969

HAL is a multi-disciplinary open access archive for the deposit and dissemination of scientific research documents, whether they are published or not. The documents may come from teaching and research institutions in France or abroad, or from public or private research centers.
L'archive ouverte pluridisciplinaire HAL, est destinée au dépôt et à la diffusion de documents scientifiques de niveau recherche, publiés ou non, émanant des établissements d'enseignement et de recherche français ou étrangers, des laboratoires publics ou privés. 


\title{
LE JOURNAL DE PHYSIQUE
}

\section{RELATIONS PARGOURS-ÉNERGIE DES IONS LOURDS DE FAIBLE VITESSE DANS LES DÉTEGTEURS VISUELS PLASTIQUES ÉTUDE PARTICULIÈRE DES FRAGMENTS DE FISSION}

\author{
Par J. RALAROSY, R. SGHMITT (1), G. MOSINSKI, J. TRIPIER, \\ M. DEBEAUVAIS, R. STEIN et G. RÉMY, \\ Département de Physique Corpusculaire, Centre de Recherches Nucléaires, Strasbourg. \\ (Reçu le 28 juin 1968, révisé le 10 septembre 1968.)
}

\begin{abstract}
Résumé. - Afin d'étudier la cinématique et le mécanisme de la fission à haute énergie, nous avons utilisé deux sortes de détecteurs visuels plastiques : le nitrate de cellulose et le makrofol. Cette étude nécessitant la connaissance de relations analytiques reliant la quantité de mouvement (ou l'énergie), la masse et le parcours des fragments dans le détecteur utilisé, nous nous sommes attachés à la détermination théorique et à la vérification expérimentale de ces relations.

Nous donnons ici une méthode de calcul permettant de déterminer le parcours d'un ion en fonction de sa vitesse dans un détecteur plastique. En partant de cette méthode et du parcours expérimental des particules $\alpha$, nous déduisons les courbes représentant la variation du parcours en fonction de la quantité de mouvement (ou de l'énergie) des ions plus lourds que les particules $\alpha$. Une étude particulière est consacrée aux fragments de fission pour lesquels des relations mathématiques simples sont données. Nous n'avons pas manqué de faire des recoupements expérimentaux avec des ions de masses et d'énergies connues.
\end{abstract}

Abstract. - To study the kinematic and mechanism of fission at high energy, we have used two kinds of plastic visual detectors, namely cellulose nitrate and makrofol. These studies require the knowledge of analytical relations between the particle momentum (or energy) and the particle range in the plastic we have used. First we have determined theoretical relations and made experimental verification.

We present here a calculation method giving particle range versus velocity in plastic detectors. These relations and the knowledge of experimental $\alpha$-particle range lead us to momentum range relations for ions heavier than $\alpha$ particles. A special calculation concerning fission fragments leads to simple mathematical relations; all necessary experimental fits have been made.

I. Considérations générales. - 1 . Historique. Les premiers travaux de Silk et Barnes [1] et de Price et Walker [2] ont montré que l'on peut observer au microscope optique des traces d'ions lourds dans certains solides isolants après une attaque chimique appropriée [3], [4], [5]. Ces détecteurs ionographiques sont d'un grand intérêt du fait de la diversité de leurs seuils d'enregistrement (dépendant en très large partie de la perte spécifique d'énergie des ions dans le matériau [4], [5], [6]). Les plus couramment utilisés sont le nitrate de cellulose $\left(\mathrm{C}_{6} \mathrm{H}_{2} \mathrm{O}_{9} \mathrm{~N}_{2}\right) n$ et le makrofol (polycarbonate de 44'-dioxydiphényl-2.2 propane $\left.\left(\mathrm{C}_{16} \mathrm{H}_{14} \mathrm{O}_{3}\right) n\right)$ dont les seuils approximatifs d'enregis-

(1) R. Schmitt, Professeur à la Faculté des Sciences de Lyon. trement correspondent respectivement aux pertes spécifiques d'énergie d'une particule $\alpha$ de $3,3 \mathrm{MeV}$ et d'un ion oxygène de $40 \mathrm{MeV}$ [7], [8].

Le regain d'intérêt apporté actuellement à l'étude du noyau, et, en particulier, à l'étude de la fission et de la fragmentation des noyaux lourds à haute énergie, nous a poussés à faire la méthodologie de ce type de détecteurs. Leur utilisation dans l'étude cinématique de la fission et de la fragmentation à haute énergie devrait nous permettre de préciser les mécanismes de ces interactions, actuellement très discutés. A cette fin, il est nécessaire d'établir, en premier lieu, des relations liant les parcours des ions à leurs énergies.

2. Notes SUR LA THÉORIE DU PASSAGe DES PARTIGULES IONISANTES DANS LA MATIÈRE. - Dans l'étude 
des relations parcours-énergie et perte spécifique d'énergie des ions lourds, on peut distinguer trois cas :

- La particule a une grande vitesse;

- La particule a une vitesse voisine de $V_{1}=v_{0} Z^{2 / 3}$ ( où $v_{0}=\frac{e^{2}}{h}=2,19 \times 10^{8} \mathrm{~cm} / \mathrm{s}$ est la vitesse de Bohr, $Z$ le nombre de charge de la particule);

- La particule a une très faible vitesse $v \simeq v_{0}[9]$.

Plusieurs hypothèses ont été émises concernant le mécanisme de formation des traces et le mode de perte d'énergie dans les détecteurs visuels plastiques [10 à 14], [15]. Quelle que soit l'hypothèse admise, retenons simplement que la perte d'énergie de la particule incidente dans la matière se fait essentiellement par interactions sur les électrons et, à un degré moindre, par interaction noyau sur noyau.

Soit :

$$
J_{\mathrm{e}}=-\left(\frac{\mathrm{d} E}{\mathrm{~d} R}\right)_{\mathrm{e}} \text { et } J_{\mathrm{n}}=-\left(\frac{\mathrm{d} E}{\mathrm{~d} R}\right)_{\mathrm{n}}
$$

respectivement la perte spécifique d'énergie par chocs électroniques et par chocs nucléaires.

D'après H. A. Bethe et U. Ashkin [16] :

$$
\eta=\frac{J_{\mathrm{n}}}{J_{\mathrm{e}}}=z\left(\frac{m}{M}\right)\left(\frac{Z}{Z_{\mathrm{eff}}}\right)^{2}=z\left(\frac{m}{M_{\mathrm{p}}}\right) \frac{1}{A}\left(\frac{Z}{Z_{\mathrm{eff}}}\right)^{2}
$$

$z$ : nombre d'électrons par atome du détecteur, $m$ : masse de l'électron,

$M$ : masse atomique moyenne du détecteur,

$M_{\mathrm{p}}$ : masse du proton,

$A$ : nombre atomique du détecteur,

$Z$ : nombre de charge de la particule incidente, $Z_{\text {eff }}$ : charge efficace de la particule incidente.

$Z_{\text {eff }}$ décroît rapidement en fin de parcours, $\eta=J_{\mathrm{n}} / J_{\mathrm{e}}$ croît en conséquence.

Choisissons la valeur proposée par Thomas Fermi [9], [18], [29] :

$$
Z_{\text {eff }}=Z^{1 / 3} \frac{\bar{h}}{e^{2}} v=137 Z^{1 / 3} \quad \text { où } \quad v=\beta c
$$

$v$ étant la vitesse de l'ion, $c$ la vitesse de la lumière.

Dans le makrofol, on aura :

$$
\eta=1,53 \times 10^{-8} Z^{4 / 3} / \beta^{2} .
$$

Le calcul montre que dans le cas d'une particule $\alpha$, $\eta$ est toujours très faible et l'on peut pratiquement négliger $J_{\mathrm{n}}$ devant $J_{\mathrm{e}}$; alors que pour des ions lourds de faible vitesse, $J_{\mathrm{n}}$ et $J_{\mathrm{e}}$ sont du même ordre en fin de parcours.

Dans le calcul du parcours en fonction de l'énergie, en négligeant $J_{\mathrm{n}}$ devant $J_{\mathrm{e}}$, on commet une erreur négligeable devant les incertitudes expérimentales. Le calcul montre que pour un ion de fission de $65 \mathrm{MeV}$ la différence entre le parcours déduit en négligeant $J_{\mathrm{n}}$ et celui déduit de $J_{\mathrm{T}}=J_{\mathrm{e}}+J_{\mathrm{n}}$ est inférieure à $0,1 \mu \mathrm{m}$ pour un parcours enregistré de l'ordre de $16,5 \mu \mathrm{m}$.

II. Relations parcours-énergie. - 1. GÉNÉRALITÉs. Méthode. But. - Pour établir les relations parcours-énergie, nous avons utilisé la relation générale [18], [29], permettant, par intégration, le calcul du parcours des ions à partir du parcours des protons. Un artifice de calcul nous permettra de déduire les parcours des ions lourds de la connaissance du parcours des particules $\alpha$, plus facilement accessible à l'expérience que le parcours des protons.

Ces calculs portant sur des ions de faible vitesse, les limites de précision que nous nous sommes imposées ont nécessité la connaissance des « extensions de parcours ». Nous y avons accédé en adaptant aux polymères les résultats proposés par Barkas pour les émulsions [18].

L'utilisation d'ions lourds tels que ${ }_{53}^{127} \mathrm{I},{ }_{16}^{32} \mathrm{~S}$ et ${ }_{8}^{16} \mathrm{O}$ produits par des accélérateurs, associée à l'étude de la fission de l'uranium par neutrons thermiques, a rendu possibles l'adaptation et l'ajustement des résultats de Barkas avec une bonne précision.

2. MÉthode DE GAlqul. - a) Relation de BraggKleemann. - On peut aborder le problème des relations parcours-énergie des ions lourds dans la matière de plusieurs façons. Le choix de la méthode ne dépend que de la précision désirée et de la gamme d'énergie dans laquelle on se propose de travailler. Comme nous nous intéressons plus spécialement aux ions de faible vitesse, une assez bonne précision nous est nécessaire.

La règle de Bragg-Kleemann [17] nous permet de comparer grossièrement (à $15 \%$ près) les parcours d'un ion dans différents matériaux. Si $R$ est le parcours de l'ion, $\rho$ la masse volumique du détecteur et $A$ sa masse molaire moyenne, nous avons la relation suivante :

$$
\frac{R_{1}}{R_{2}}=\frac{\rho_{2}}{\rho_{1}} \times \sqrt{A_{1} / A_{2}}
$$

les indices 1 et 2 étant relatifs aux deux matériaux considérés.

Si $R_{\text {mak }}, R_{\text {nit }}$ et $R_{\text {ém }}$ sont respectivement les parcours d'un ion dans le makrofol, le nitrate de cellulose et l'émulsion nucléaire standard, le calcul nous donne :

$$
\begin{aligned}
& \frac{R_{\mathrm{mak}}}{R_{\mathrm{nit}}}=1,24 \pm 0,18 \\
& \frac{R_{\mathrm{mak}}}{R_{\text {ém }}}=1,63 \pm 0,24 \\
& \frac{R_{\text {nit }}}{R_{\text {èm }}}=1,3 \pm 0,2
\end{aligned}
$$

les masses volumiques étant respectivement de $\rho=1,2 \mathrm{~g} / \mathrm{cm}^{3}$ pour le makrofol et de $\rho=1,6 \mathrm{~g} / \mathrm{cm}^{3}$ pour le nitrate de cellulose utilisé. 
b) Relation parcours-vitesse des ions. - L'obtention des relations parcours-énergie à partir de la détermination du pouvoir d'arrêt différentiel, puis intégré, nécessite la connaissance précise du potentiel moyen d'ionisation et du terme correctif dû à l'effet d'écran. Nous préférons utiliser une méthode semi-théorique basée sur l'intégration de l'ionisation spécifique $\mathrm{d} E / \mathrm{d} R$ [18], [29] en nous référant aux parcours expérimentaux des particules $\alpha$ que nous aurons nous-mêmes déterminés expérimentalement pour chacun de nos détecteurs.

Pour une particule de vitesse $\beta c$, de nombre de charge $Z$ et de masse $M$, le parcours $R$ est donné par la relation :

$$
R=\frac{M}{Z^{2}} R_{\mathrm{p}}(\beta)+R_{\text {ext }}(\beta)
$$

$R_{\mathrm{p}}(\beta)$ est le parcours du proton de vitesse $\beta c$ dans le matériau considéré,

$R_{\text {ext }}(\beta)$ est l'extension de parcours due à la neutralisation des charges de la particule incidente par le matériau traversé.

Pour tenter de donner une définition subjective grossière de l'extension de parcours, on remarquera qu'à même énergie cinétique le parcours d'une particule dans la matière est d'autant plus grand que sa charge est plus faible (c'est-à-dire que son freinage est moindre).

Si l'on détermine le parcours par l'intégrale :

$$
R=\int_{E=E_{0}}^{E=0}\left(\frac{\mathrm{d} E}{\mathrm{~d} E / \mathrm{d} R}\right)
$$

(où $\mathrm{d} E / \mathrm{d} R$ est donné, par exemple, par la formule de Bethe [18]), il faut tenir compte du fait qu'en fin de parcours, lorsque la vitesse de l'ion voisine celle des électrons périphériques, la charge moyenne de l'ion diminue progressivement par capture électronique. Cela se traduit par une diminution de la charge efficace $Z_{\text {eff. }}$ On est ramené dans cette zone de faible vitesse à sommer des éléments de parcours correspondant à différentes valeurs de la charge $Z$ (on sommera de $Z=Z_{\text {eff }}$ à $Z=0$ lorsque la vitesse tend vers zéro). En fait, on montre que $Z_{\text {eff }}$ est une fonction de la vitesse seule.

Physiquement, tout se passe comme si la décroissance de $Z_{\text {eff }}$ en fin de parcours entraînait une majoration du parcours calculé par l'intégration de $\mathrm{d} E / \mathrm{d} R$ avec $Z_{\text {eff }}$ constante. Cette majoration représente l'extension de parcours. Elle a pour origine l'existence aux faibles vitesses d'éléments de parcours supérieurs à ceux prévus par l'intégrale, la charge, donc le freinage, ayant diminué dans cette zone.

Pour une particule $\alpha, M / Z^{2}=1$ et son parcours est donné par la relation :

$$
R_{\alpha}=R_{\mathrm{p}}(\beta)+R_{\text {ext }}^{\alpha}(\beta)
$$

où $R_{\text {ext }}^{\alpha}$ est l'extension de parcours de la particule $\alpha$. On en tire :

$$
R_{\mathrm{p}}(\beta)=R_{\alpha}-R_{\mathrm{ext}}^{\alpha} .
$$

Donc, pour un ion de charge $Z$ et de masse $M$ :

$$
R=\frac{M}{Z^{2}}\left(R_{\alpha}-R_{\mathrm{ext}}^{\alpha}\right)+R_{\mathrm{ext}}(\beta) .
$$

c) Détermination de l'extension de parcours $R_{\text {ext }}$. Posons :

$$
R_{\mathrm{ext}}=\frac{M}{Z^{2}} B_{Z}(\beta)
$$

On sait que [18] :

$$
B_{Z}\left(\lambda_{1}\right)=\int_{0}^{\lambda_{1}}\left[\left(\frac{Z}{Z_{\text {eff }}}\right)^{2}-1\right] \mathrm{d} \lambda
$$

$Z_{\text {eff }}:$ charge efficace de la particule,

$\lambda$ : parcours idéal du proton dans le matériau considéré.

D'après les résultats compilés par Whaling (cité par W. H. Barkas [18]), la fonction $Z_{\text {eff }} / Z$ ne dépend que de $\beta / Z^{2 / 3}$ et est indépendante du matériau traversé.

$\left(Z / Z_{\text {eff }}\right)^{2}-1$ étant une fonction monotone de $\beta / Z^{2 / 3}$, on peut écrire :

$$
B_{Z}\left(\lambda_{1}\right)=\left\langle\left(\frac{Z}{Z_{\text {eff }}}\right)^{2}-1\right\rangle \int_{0}^{\lambda_{1}} \mathrm{~d} \lambda
$$

$(<>$ indique la valeur moyenne).

Dans le cas des émulsions nucléaires standard, Barkas [18] a représenté graphiquement la fonction :

$$
C_{Z}=\frac{B_{Z}(\beta)}{Z^{8 / 3}}
$$

C'est une fonction monotone croissante de $137 \frac{\beta}{Z}$. Sa variation est à peu près linéaire pour $\frac{137 \beta}{Z}<2$; elle tend vers une valeur asymptotique pour $\frac{137 \beta}{Z}>2$; elle est nulle pour $\beta=0$.

Heckmann et coll. [19] ont donné la valeur asymptotique suivante dans le cas de l'émulsion nucléaire :

$$
B_{Z \text { asympt. }}=0,2 \times Z^{2,72} \text { en } \mu \mathrm{m} \text {. }
$$

D'après l'expression de $B_{Z}\left(\lambda_{1}\right)$, le rapport des deux fonctions $C_{Z}(\mathrm{a})$ et $C_{Z}(\mathrm{e})$ relatives à un matériau (a) et à l'émulsion (e) est donné par :

$$
C_{Z}^{(\mathrm{a})} / C_{Z}^{(\mathrm{e})} \simeq \lambda^{\mathrm{a}} / \lambda^{\mathrm{e}}
$$

où $\lambda^{a}$ et $\lambda^{e}$ représentent respectivement le parcours idéal du proton dans le détecteur (a) et dans l'émulsion (e), pour le domaine où $Z_{\text {eff }} \leqslant Z$.

En première approximation, utilisant la règle de Bragg-Kleemann, nous avons, pour le makrofol :

$$
\lambda^{(a)} / \lambda^{(e)} \simeq 1,6 \text {. }
$$

Compte tenu de ce rapport, nous avons, à partir de la courbe de Barkas, représenté graphiquement la variation de l'extension de parcours, relative à chacune des particules qui nous intéressent en fonction de $\beta / Z$. De légères corrections ont été apportées, en particulier 
pour les ions très lourds de faible vitesse. Ces corrections ont permis d'ajuster les valeurs théoriques aux valeurs expérimentales trouvées.

d) Détermination expérimentale $d u$ parcours $R_{\alpha}$ d'une particule $\alpha$ en fonction de son énergie cinétique. - Pour ces mesures, nous utilisons une boîte à cible cylindrique comprenant :

- Une source $\mathrm{S}$ de rayons $\alpha$ disposée derrière un obturateur $\mathrm{O}$. On utilise une source de polonium comme source $\alpha$;

- Un porte-détecteur $\mathrm{D}$, d'inclinaison variable, mobile autour d'une droite perpendiculaire à l'axe du cylindre;

- Un porte-absorbant A, dans lequel on peut disposer une série de couronnes circulaires portant, bien tendue à l'intérieur, une membrane de matière plastique d'épaisseur différente (makrofol ou nitrate de cellulose) servant d'absorbant.

Le vide est, à l'intérieur du cylindre, de l'ordre de $10^{-3}$ torr. L'utilisation de diaphragmes nous permet d'avoir un faisceau parallèle et d'opérer en « bonne géométrie ».

Le détecteur est constitué d'une plaque de verre rectangulaire dont une moitié est couverte d'une émulsion $\mathrm{K} 5$ de $50 \mu \mathrm{m}$ et l'autre moitié d'une feuille du détecteur plastique considéré. L'émulsion servira d'étalon dans les mesures d'énergie.

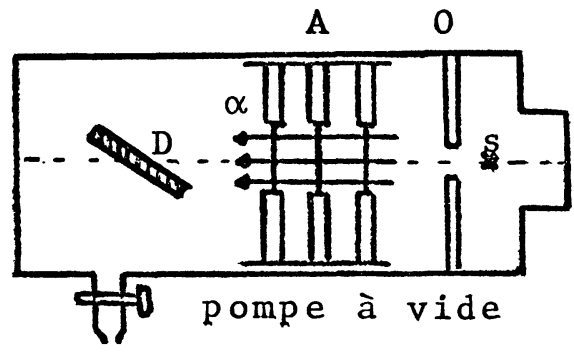

Au départ, on n'interpose aucun absorbant dans le faisceau. L'énergie des particules $\alpha$ utilisées est de $5,3 \mathrm{MeV}$, on vérifie cette valeur par la mesure du parcours moyen dans l'émulsion. Aucune trace n'apparaît dans le nitrate de cellulose ou le makrofol après un développement standard habituel à la soude. En effet, les matériaux plastiques utilisés étant des détecteurs à seuil, la perte d'énergie nécessaire pour créer des traces visibles n'est assez élevée qu'au-dessus d'une valeur seuil; cette valeur correspond à $3,3 \mathrm{MeV}$ pour une particule $\alpha$ dans le nitrate de cellulose [6]. Autrement dit, seules des particules alpha, d'énergie inférieure à $3,3 \mathrm{eV}$ et possédant de ce fait une ionisation spécifique suffisante, seront enregistrées [8].

Les absorbants sont ensuite placés devant la source par épaisseur croissante. A chaque épaisseur d'absorbant sera associé un ensemble de détecteurs (émulsion et plastique) dans lequel on mesurera la longueur moyenne des traces. Les mesures de parcours effectuées dans l'émulsion nous donneront l'énergie des $\alpha$ recueillies après traversée de la série d'absorbants. On aura donc, et le parcours des $\alpha$ dans le détecteur plastique, et l'énergie des particules $\alpha$. Dans le cas des détecteurs insensibles aux $\alpha$ tel le makrofol, ou pour des énergies supérieures à l'énergie seuil, on se contente de mesurer l'épaisseur d'absorbant et, à l'aide des émulsions, la perte d'énergie pour cette épaisseur d'absorbant.

On représente graphiquement la variation de l'énergie en fonction de l'épaisseur de l'absorbant. On en déduit la courbe parcours-énergie dans le matériau plastique considéré. Les figures 1 et 2 montrent respec-

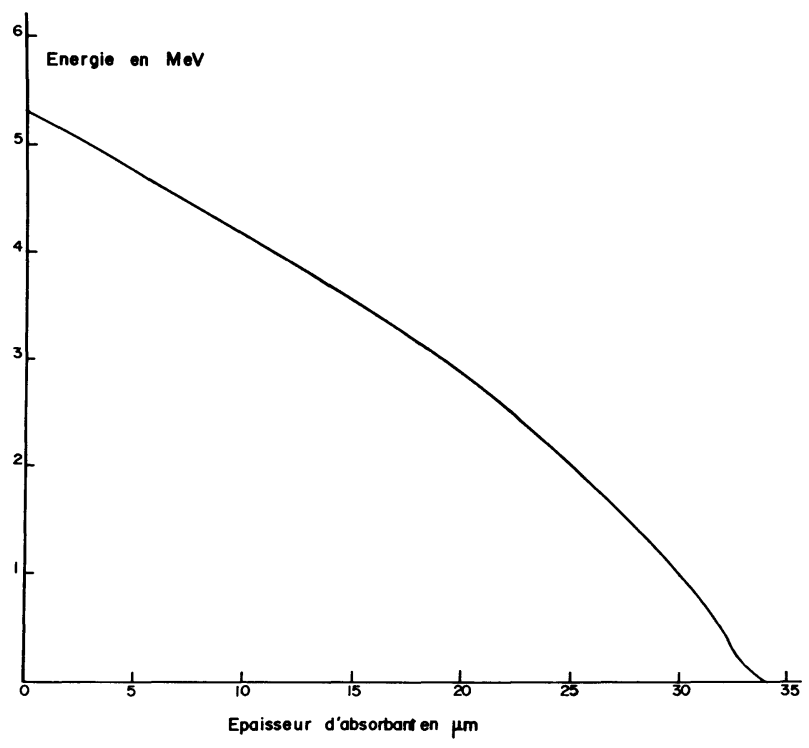

FIG. 1. - Relation parcours-énergie des particules alpha dans le makrofol.

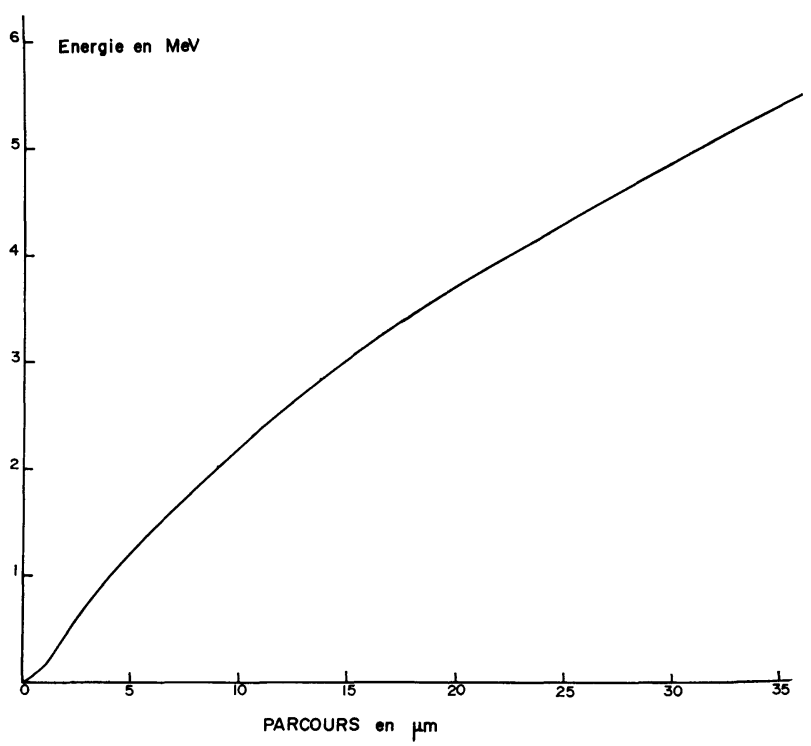

FIG. 2. - Relation parcours-énergie des particules alpha dans le makrofol. 
TABLEAU I

RELATION PARGOURS-ÉNERGIE DANS LE MAKROFOL

\begin{tabular}{|c|c|c|c|c|c|c|c|c|c|c|}
\hline \multirow{2}{*}{$\begin{array}{c}\text { ÉNERGIE } \\
\text { EN } \\
\mathrm{MeV} / \text { nucléon }\end{array}$} & \multicolumn{10}{|c|}{ PARCOURS EN $\mu \mathrm{m}$} \\
\hline & ${ }_{2}^{4} \mathrm{He}$ & ${ }_{3}^{6} \mathrm{~L}_{\mathrm{i}}$ & ${ }_{3}^{8} \mathrm{Li}$ & ${ }_{4}^{9} \mathrm{Be}$ & ${ }_{5}^{10} \mathrm{~B}$ & ${ }_{6}^{12} \mathrm{C}$ & ${ }_{7}^{14} \mathrm{~N}$ & ${ }_{8}^{16} \mathrm{O}$ & ${ }_{11}^{22} \mathrm{Na}$ & ${ }_{13}^{27} \mathrm{~A} 1$ \\
\hline - & - & - & - & - & - & - & - & - & - & - \\
\hline 0,05 & 1,20 & 1,20 & 1,65 & 1,50 & 1,35 & 1,50 & 1,50 & 1,40 & 1,25 & 1,30 \\
\hline 0,10 & 1,85 & 1,90 & 2,55 & 2,35 & 2,20 & 2,35 & 2,45 & 2,45 & 2,60 & 2,80 \\
\hline 0,15 & 2,50 & 2,50 & 3,45 & 3,00 & 2,85 & 3,05 & 3,20 & 3,30 & 3,65 & 3,95 \\
\hline 0,20 & 3,25 & 3,15 & 4,30 & 3,75 & 3,45 & 3,70 & 3,85 & 3,95 & 4,50 & 4,90 \\
\hline 0,25 & 4,05 & 3,90 & 5,25 & 4,45 & 4,10 & 4,30 & 4,45 & 4,55 & 5,20 & 5,70 \\
\hline 0,30 & 4,90 & 4,65 & 6,20 & 5,20 & 4,75 & 4,90 & 5,05 & 5,20 & 5,85 & 6,45 \\
\hline 0,35 & 5,80 & 5,50 & 7,25 & 5,95 & 5,40 & 5,50 & 5,65 & 5,80 & 6,50 & 7,15 \\
\hline 0,40 & 6,80 & 6,35 & 8,30 & 6,75 & 6,05 & 6,10 & 6,25 & 6,40 & 7,10 & 7,90 \\
\hline 0,45 & 7,80 & 7,15 & 9,45 & 7,55 & 6,75 & 6,75 & 6,85 & 7,00 & 7,70 & 8,65 \\
\hline 0,50 & 8,90 & 8,00 & 10,50 & 8,40 & 7,45 & 7,40 & 7,50 & 7,65 & 8,30 & 9,40 \\
\hline 0,55 & 10,00 & 8,90 & 11,75 & 9,25 & 8,15 & 8,10 & 8,15 & 8,25 & 8,85 & 10,05 \\
\hline 0,60 & 11,10 & 9,80 & 13,00 & 10,15 & 8,90 & 8,80 & 8,85 & 8,90 & 9,40 & 10,70 \\
\hline 0,65 & 12,30 & 10,65 & 14,25 & 11,05 & 9,60 & 9,50 & 9,50 & 9,55 & 10,00 & 11,40 \\
\hline 0,70 & 13,50 & 11,50 & 15,55 & 11,95 & 10,30 & 10,20 & 10,15 & 10,20 & 10,65 & 12,10 \\
\hline 0,75 & 14,80 & 10,45 & 16,75 & 12,90 & 11,05 & 10,90 & 10,80 & 10,90 & 11,25 & 12,80 \\
\hline 0,80 & 16,15 & 13,45 & 17,95 & 13,85 & 11,80 & 11,60 & 11,50 & 11,60 & 11,90 & 13,50 \\
\hline 0,85 & 17,65 & 14,45 & 19,40 & 14,90 & 12,55 & 12,35 & 12,20 & 12,25 & 12,50 & 14,25 \\
\hline 0,90 & 19,20 & 15,50 & 20,80 & 15,95 & 13,35 & 13,10 & 12,90 & 12,90 & 13,10 & 15,00 \\
\hline 0,95 & 20,80 & 16,60 & 22,25 & 17,05 & 14,10 & 13,85 & 13,60 & 13,55 & 13,70 & 15,65 \\
\hline 1,00 & 22,50 & 17,75 & 23,75 & 18,15 & 14,90 & 14,65 & 14,30 & 14,25 & 14,35 & 16,35 \\
\hline 1,05 & 24,25 & 18,85 & 25,25 & 19,30 & 15,75 & 14,40 & 15,05 & 14,90 & 15,00 & 17,05 \\
\hline 1,10 & 25,90 & 20,00 & 26,80 & 20,45 & 16,65 & 16,20 & 15,80 & 15,60 & 15,65 & 17,80 \\
\hline 1,15 & 27,60 & 21,25 & 28,35 & 21,60 & 17,55 & 16,95 & 16,55 & 16,35 & 16,30 & 18,50 \\
\hline 1,20 & 29,40 & 22,50 & 29,90 & 22,75 & 18,50 & 17,75 & 17,30 & 17,10 & 16,95 & 19,20 \\
\hline 1,25 & 31,10 & 23,75 & 31,55 & 23,90 & 19,40 & 18,50 & 18,00 & 17,75 & 17,55 & 19,80 \\
\hline 1,30 & 33,15 & 25,00 & 33,10 & 25,05 & 20,35 & 19,25 & 18,75 & 18,40 & 18,15 & 20,40 \\
\hline 1,35 & 35,15 & 26,25 & 34,80 & 26,20 & 21,25 & 20,00 & 19,50 & 19,10 & 18,75 & 21,05 \\
\hline 1,40 & 36,90 & 27,50 & 36,50 & 27,40 & 22,20 & 20,80 & 20,25 & 19,80 & 19,40 & 21,70 \\
\hline 2,00 & 66,00 & 46,80 & 62,45 & 42,90 & 35,20 & 35,50 & 30,50 & 29,50 & 27,70 & 27,60 \\
\hline
\end{tabular}

tivement les variations de l'énergie en fonction de l'épaisseur d'absorbant et la courbe parcours-énergie. On peut, avec une bonne approximation, extrapoler ces courbes jusqu'à une énergie des particules alpha de l'ordre de $8 \mathrm{MeV}$.

e) Détermination du parcours $R$ d'un ion lourd en fonction de son énergie cinétique. - De la mesure expérimentale du parcours $R_{\alpha}$ des particules $\alpha$ et du calcul des extensions de parcours $R_{\mathrm{ext}}^{\alpha}$ et $R_{\mathrm{ext}}^{\mathrm{ion}}(\beta)$ pour une vitesse $\beta$ donnée, correspondant respectivement au parcours de la particule $\alpha$ et à celui de l'ion de nombre de charge $Z$ de même vitesse $\beta$, on peut, à l'aide de la formule $R=\left[M / Z^{2}\right]\left[R_{\alpha}(\beta)-R_{\text {ext }}^{\alpha}(\beta)\right]+R_{\text {ext }}^{\text {ion }}(\beta)$, déduire, pour le makrofol par exemple, les tableaux I et II donnant le parcours des ions de nombre de charge $Z$ et de masse $M$ en fonction de leur énergie.

Pratiquement, si on se donne l'énergie cinétique $E$ de l'ion de nombre de charge $Z$ et de masse $M$, le calcul fournit sa quantité de mouvement et sa vitesse.

On peut donc, des mesures de $R_{\alpha}$ et du calcul de $R_{\text {ext }}^{\alpha}$, déduire le parcours et l'extension de parcours d'une particule $\alpha$ ayant la même vitesse $\beta$ que l'ion étudié. L'extension de parcours $R_{\mathrm{ext}}^{\text {ion }}(\beta)$ correspondant

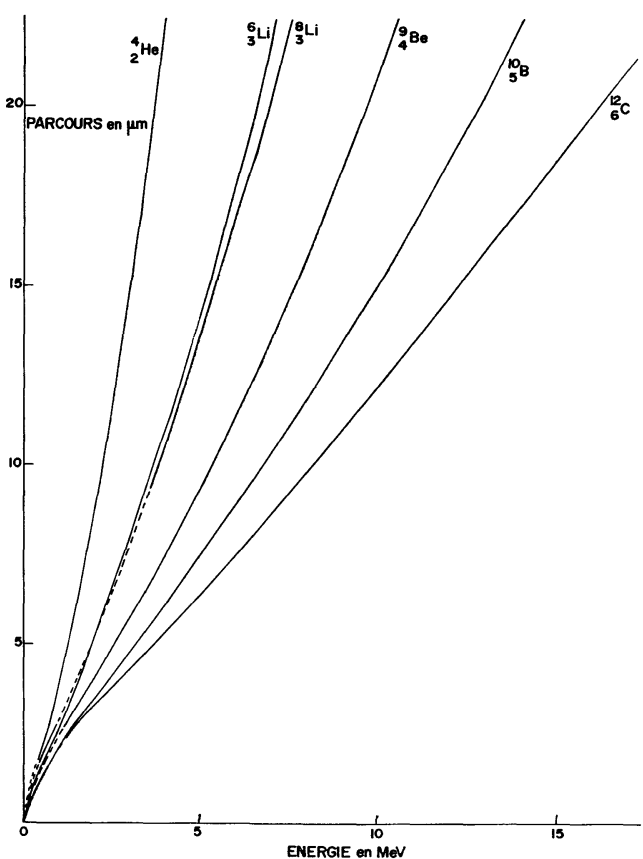

FIG. 3. - Courbes parcours-énergie dans le makrofol. 
TABLEAU II

RELATION PARGOURS-ÉNERGIE DANS LE MAKROFOL

\begin{tabular}{|c|c|c|c|c|c|c|c|c|c|c|}
\hline \multirow{2}{*}{$\begin{array}{l}\text { ÉNERGIE } \\
\text { EN } \\
\mathrm{MeV} / \text { nucléon }\end{array}$} & \multicolumn{10}{|c|}{ PARCOURS EN $\mu \mathrm{m}$} \\
\hline & ${ }_{14}^{29} \mathrm{Si}$ & ${ }_{15}^{31} \mathrm{P}$ & ${ }_{16}^{32} \mathrm{~S}$ & ${ }_{20}^{43} \mathrm{Ca}$ & ${ }_{26}^{56} \mathrm{Fe}$ & ${ }_{29}^{65} \mathrm{Cu}$ & ${ }_{34}^{79} \mathrm{Se}$ & ${ }_{38}^{97} \mathrm{Sr}$ & ${ }_{46}^{117} \mathrm{Pd}$ & ${ }_{54}^{138} \mathrm{Xe}$ \\
\hline - & - & - & - & - & - & - & - & - & - & - \\
\hline 0,05 & 1,10 & 1,15 & 1,45 & 1,40 & 1,80 & 2,60 & 3,40 & 3,30 & 4,15 & 4,80 \\
\hline 0,10 & 2,10 & 2,15 & 2,10 & 2,70 & 3,30 & 4,40 & 5,00 & 5,60 & 6,35 & 7,20 \\
\hline 0,15 & 3,20 & 3,10 & 3,20 & 3,95 & 4,60 & 5,40 & 6,30 & 7,10 & 8,05 & 9,05 \\
\hline 0,20 & 4,25 & 4,20 & 4,30 & 5,05 & 5,80 & 6,50 & 7,50 & 8,40 & 9,50 & 10,55 \\
\hline 0,25 & 5,20 & 5,15 & 5,00 & 6,10 & 6,95 & 7,55 & 8,50 & 9,50 & 10,55 & 11,80 \\
\hline 0,30 & 6,10 & 6,05 & 6,30 & 7,00 & 7,95 & 8,60 & 9,45 & 10,50 & 11,70 & 13,00 \\
\hline 0,35 & 6,90 & 6,90 & 6,55 & 7,95 & 8,90 & 9,55 & 10,35 & 11,40 & 12,70 & 14,10 \\
\hline 0,40 & 7,70 & 7,70 & 8,30 & 8,85 & 9,85 & 10,50 & 11,20 & 12,20 & 13,65 & 15,20 \\
\hline 0,45 & 8,45 & 8,45 & 7,95 & 9,70 & 10,75 & 11,35 & 12,00 & 12,95 & 14,55 & 16,15 \\
\hline 0,50 & 9,20 & 9,25 & 9,80 & 10,50 & 11,65 & 12,20 & 12,80 & 13,70 & 15,45 & 17,05 \\
\hline 0,55 & 9,90 & 9,95 & 9,25 & 11,35 & 12,50 & 12,95 & 13,60 & 14,45 & 16,25 & 17,90 \\
\hline 0,60 & 10,60 & 10,65 & 11,00 & 12,15 & 13,30 & 13,70 & 14,35 & 15,20 & 17,00 & 18,70 \\
\hline 0,65 & 11,20 & 11,25 & 10,45 & 12,90 & 14,10 & 14,50 & 15,15 & 15,85 & 17,75 & 19,45 \\
\hline 0,70 & 11,85 & 11,90 & 12,10 & 13,65 & 14,90 & 15,25 & 15,90 & 16,50 & 18,50 & $20,2_{0}$ \\
\hline 0,75 & 12,45 & 12,55 & 11,60 & 14,40 & 15,65 & 16,00 & 16,55 & 17,10 & 19,25 & 20,95 \\
\hline 0,80 & 13,10 & 13,20 & 13,10 & 15,10 & 16,45 & 16,70 & 17,20 & 17,70 & 20,00 & 21,70 \\
\hline 0,85 & 14,65 & 13,80 & 12,90 & 15,80 & 17,15 & 15,45 & 17,90 & 18,25 & 20,70 & 22,45 \\
\hline 0,90 & 14,20 & 14,40 & 14,10 & 16,50 & 17,90 & 18,15 & 18,60 & 18,80 & 21,40 & 23,20 \\
\hline 0,95 & 14,80 & 14,95 & 14,70 & 17,20 & 18,65 & 18,85 & 19,30 & 19,35 & 22,05 & 23,95 \\
\hline 1,00 & 15,40 & 15,50 & 15,30 & 17,90 & 19,40 & 19,50 & 20,00 & 19,90 & 22,70 & 24,70 \\
\hline 1,05 & 16,00 & 16,10 & 15,75 & 18,55 & 20,05 & 20,15 & 20,65 & 20,45 & 23,35 & 25,45 \\
\hline 1,10 & 16,60 & 16,70 & 16,20 & 19,15 & 20,65 & 20,80 & 21,30 & 21,00 & 24,00 & 26,20 \\
\hline 1,15 & 17,25 & 17,30 & 16,70 & 19,80 & 21,35 & 21,50 & 21,95 & 21,55 & 24,65 & 26,95 \\
\hline 1,20 & 17,90 & 17,95 & 17,25 & 20,40 & 22,00 & 22,20 & 22,55 & 22,10 & 25,30 & 27,70 \\
\hline 1,25 & 18,50 & 18,55 & 17,80 & 21,00 & 22,60 & 22,85 & 23,15 & 22,65 & 25,95 & 28,45 \\
\hline 1,30 & 19,10 & 19,20 & 18,35 & 21,60 & 23,20 & 23,50 & 23,75 & 23,20 & 26,60 & 29,20 \\
\hline 1,35 & 19,75 & 19,85 & 18,90 & 22,15 & 23,80 & 24,15 & 24,35 & 23,75 & 27,25 & 29,95 \\
\hline 1,40 & 20,40 & 20,50 & 19,45 & 22,75 & 24,40 & 24,80 & 24,90 & 24,30 & 27,90 & 30,70 \\
\hline
\end{tabular}

à l'ion de vitesse $\beta$ est déduit de la courbe de Barkas (comme indiqué sous c).

Par application de :

$$
R=M / Z^{2}\left[R_{\alpha}(\beta)-R_{\mathrm{ext}}^{\alpha}\right]+R_{\mathrm{ext}}^{\mathrm{ion}}(\beta)
$$

on obtient le parcours cherché.

Nous avons également représenté graphiquement ( fig. 3 à 6) les variations du parcours des ions en fonction de leur énergie dans le cas du makrofol.

f) Relation parcours-énergie pour un fragment de fission. Nous avons :

$$
R=\frac{M}{Z^{2}}\left(R_{\alpha}-R_{\mathrm{ext}}^{\alpha}+B_{Z}\right)
$$

avec :

$$
B_{Z}=Z^{8 / 3} C_{Z} \text {. }
$$

Pour les fragments de vitesse assez faible, c'est-àdire pour $\frac{137 \beta}{Z} \leqslant 0,2, C_{Z}$ est une fonction linéaire de $\frac{137 \beta}{Z}$ :

$$
C_{Z}=k \frac{137 \beta}{Z}
$$

$k$ est une constante que nous nous proposons de déterminer expérimentalement.
D'après la courbe de Barkas, dans l'émulsion, on a $k_{\mathrm{e}}=0,06$ pour les fragments de fission.

Si l'on se réfère aux parcours moyens des fragments

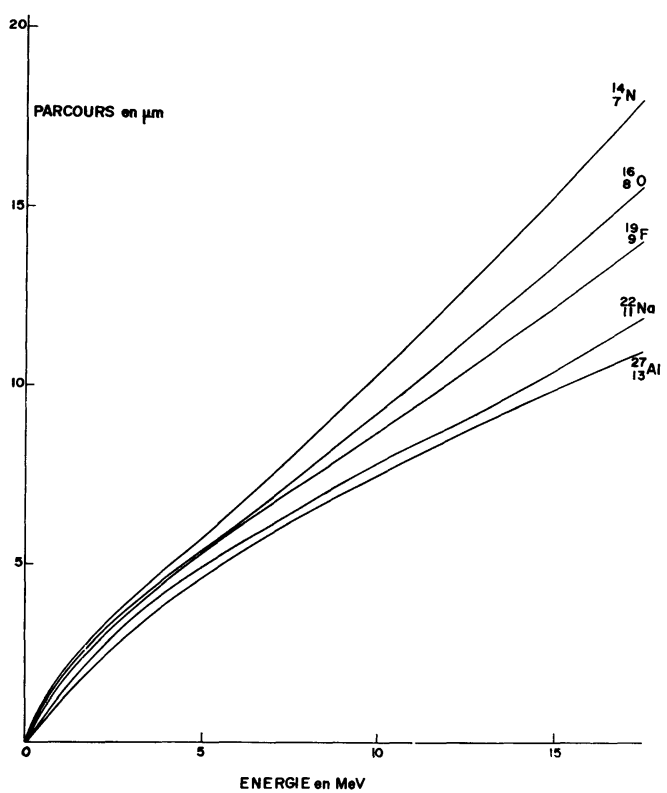

Fig. 4. - Courbes parcours-énergie dans le makrofol. 


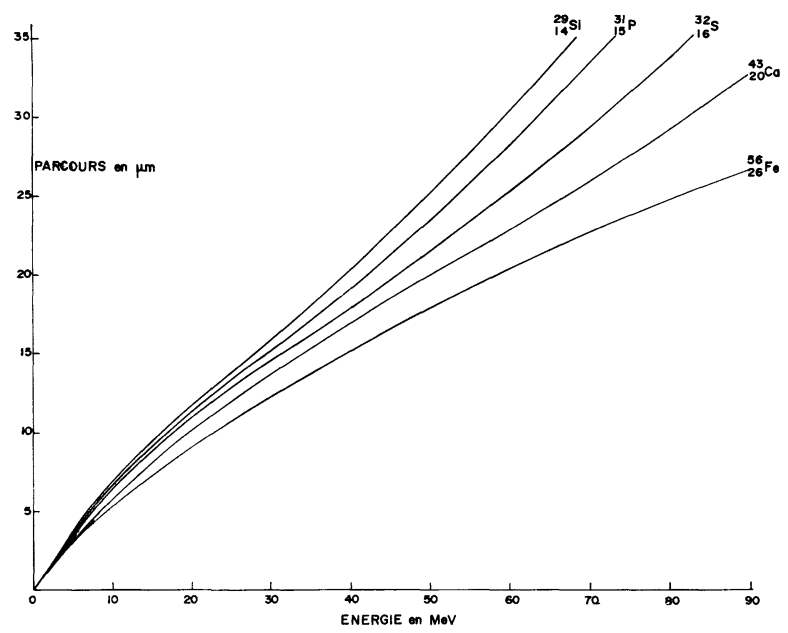

Fig. 5. - Courbes parcours-énergie dans le makrofol.

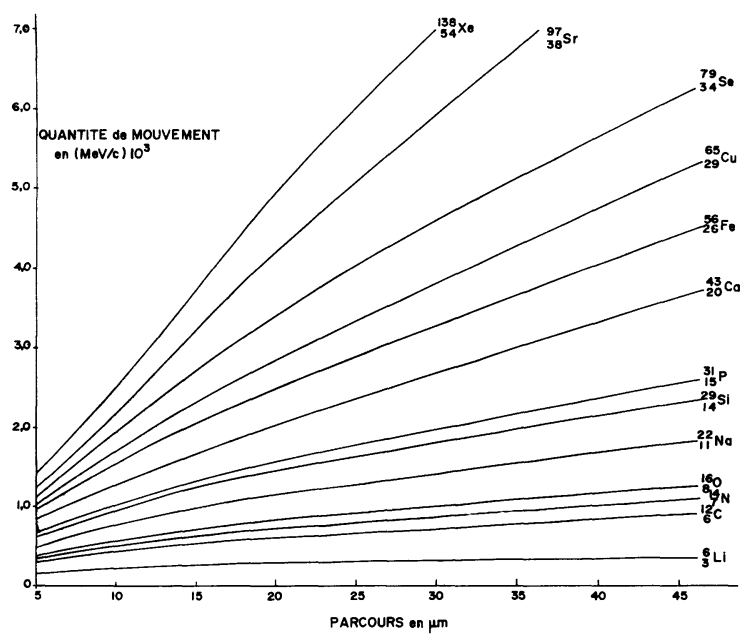

FIG. 6. - Courbes parcours-quantité de mouvement dans le makrofol.

lourds et légers de la fission de l'uranium par neutrons thermiques dans l'émulsion, la valeur de la constante $k$ est de 0,0645. Ces parcours moyens sont de 10,4 et $13,4 \mu \mathrm{m}$ [20]. Dans le makrofol, nos mesures expérimentales de parcours [21], [22] nous conduisent à la valeur $k=0,103$.

Donc :

$$
C_{Z}=0,103 \frac{137 \beta}{Z}=14,11 \frac{\beta}{Z}
$$

et il vient :

$$
R(\beta)=\frac{M}{Z^{2}}\left[R_{\alpha}(\beta)-R_{\mathrm{ext}}^{\alpha}(\beta)+14,11 Z^{5 / 3} \beta\right] .
$$

Nous avons ainsi exprimé le parcours d'un ion de masse $M$, de nombre de charge $Z$ et de vitesse $\beta c$.

Nous avons représenté sur les figures 7 à 9 les courbes parcours-énergie et parcours-quantité de mouvement correspondant aux ions de fission. g) Expressions analytiques du parcours dans le makrofol en fonction de l'énergie et de la quantité de mouvement de la particule. - Il est intéressant d'avoir une relation analytique simple entre le parcours et l'énergie ou la quantité de mouvement de la particule considérée.

Dans le cas des fragments de fission, on remarque :

1) Qu'à énergie donnée, le parcours ne varie que très faiblement avec la masse de la particule;

2) Que pour une masse donnée, cette courbe $E=f(R)$ est pratiquement parabolique, du moins dans le domaine des faibles vitesses.

Si $R$ est exprimé en $\mu \mathrm{m}$ et $E$ en $\mathrm{MeV}$, les courbes semi-expérimentales que nous avons obtenues satisfont aux équations suivantes :

Pour le ${ }_{54}^{138} \mathrm{Cs}$, on a :

$$
E=0,22 R^{2}+0,50 R \text {. }
$$

Pour le ${ }_{38}^{97} \mathrm{Sr}$, on a :

$$
E=0,22 R^{2}+0,35 R \text {. }
$$

On peut donc écrire, pour un ion de fission de masse quelconque, la relation suivante :

$$
E=0,22 R^{2}+0,42 R \text {. }
$$

L'incertitude sur l'énergie, lorsqu'on utilise cette dernière relation, est de $3 \mathrm{MeV}$ pour un parcours $R$ fixé ou de $1 \mu \mathrm{m}$ sur le parcours pour une énergie $E$ fixée.

Ces relations sont valables pour $R \lesssim 20 \mu \mathrm{m}$.

Pour les ions lourds dont la masse $M$ est comprise entre 20 et 90 , nous avons fait un lissage des courbes parcours-quantité de mouvement.

L'approximation polygonale semble le mieux convenir aux applications cinématiques en vue desquelles nous avons été contraints d'effectuer cette étude.

Si $P$ est la quantité de mouvement, la courbe $P=f(R, M)$ est approximativement linéaire dans un intervalle donné et est de la forme :

$$
P=A R+B
$$

où $A$ et $B$ sont des fonctions de la masse $M$ de la particule.

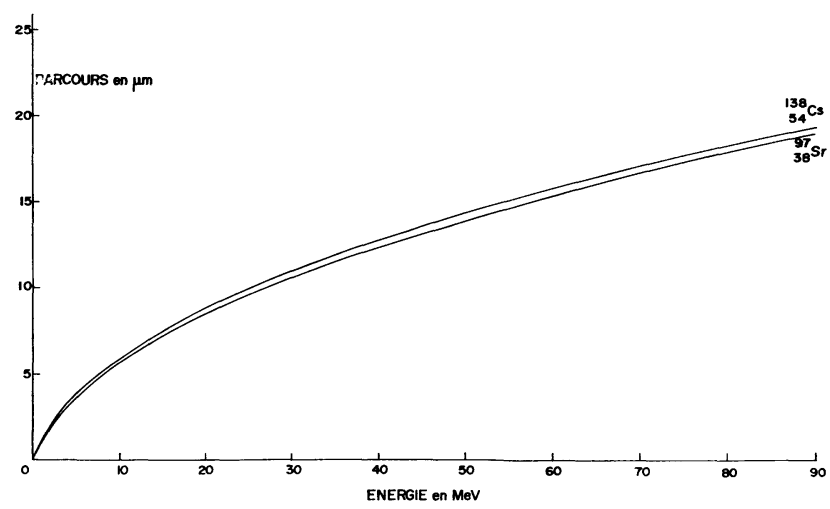

FIG. 7. - Courbes parcours-énergie des fragments de fission dans le makrofol. 


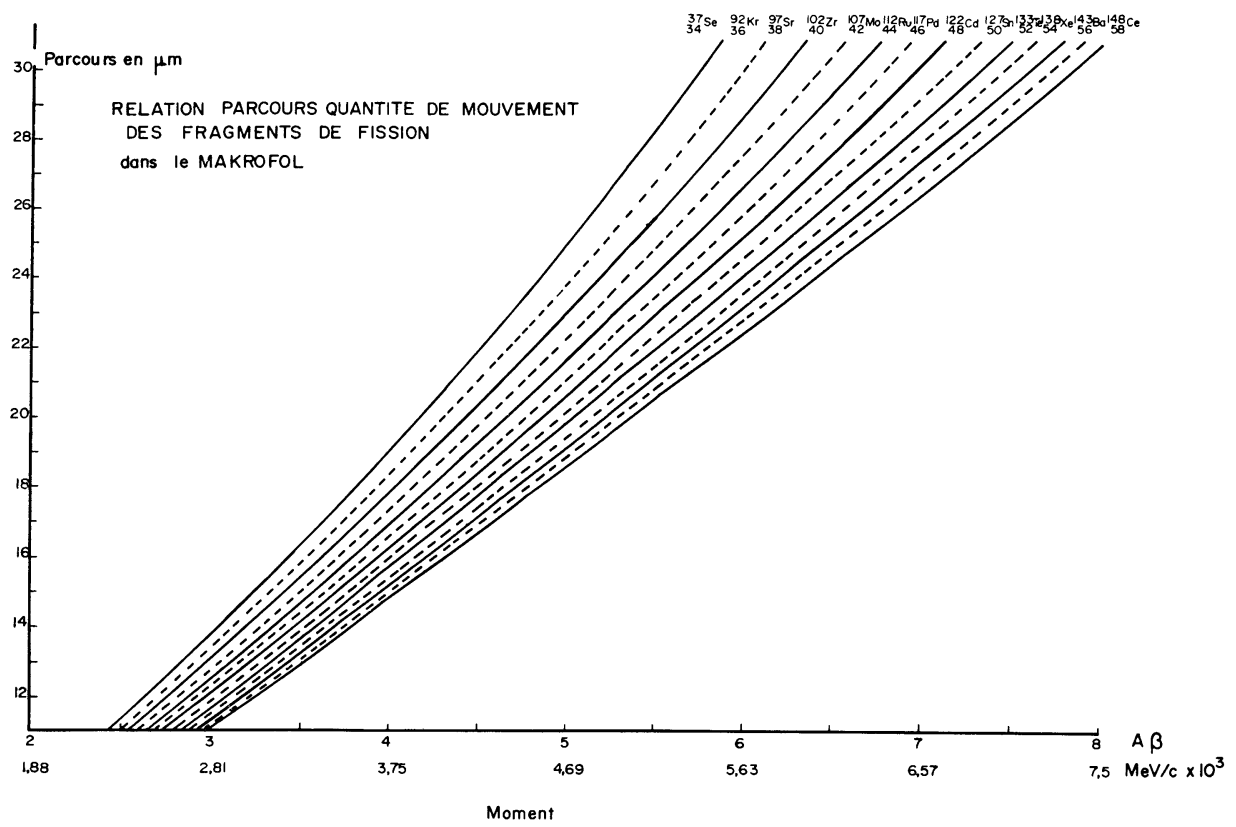

FIG. 8. - Relation parcours-quantité de mouvement des fragments de fission dans le makrofol.

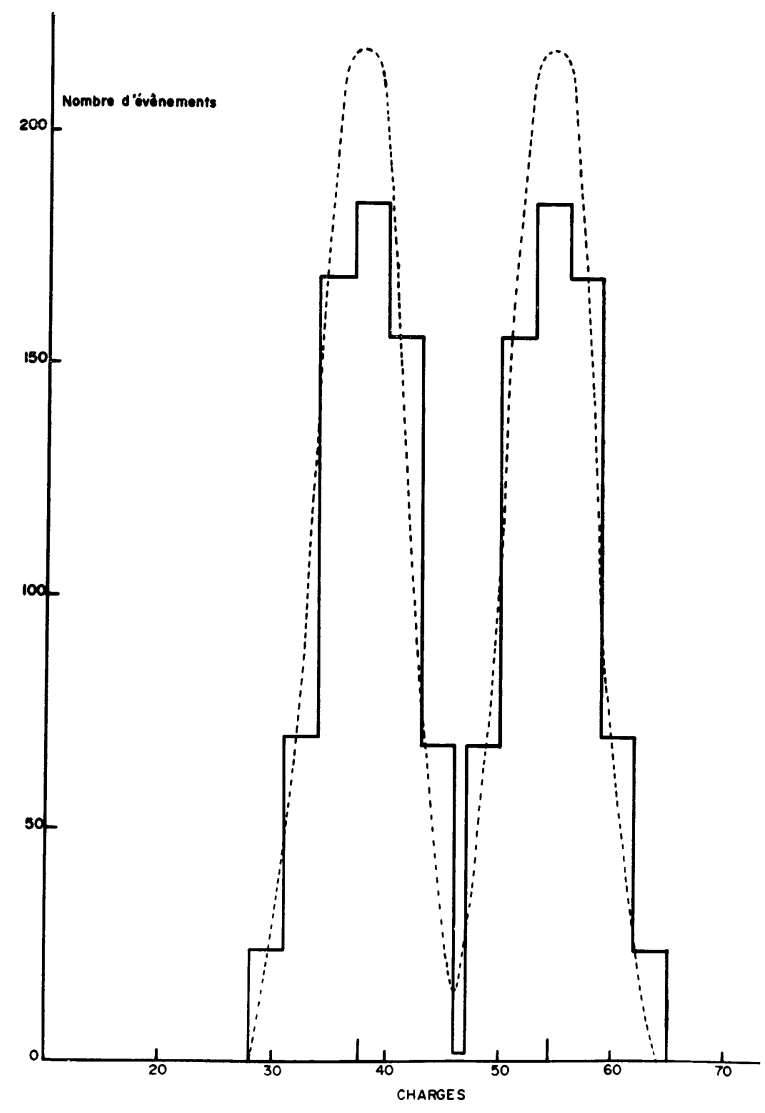

FIG. 9. - Fission de l'uranium par neutrons thermiques. Distribution des charges obtenue par la méthode des détecteurs ionographiques solides.
Si nous exprimons $P$ en $\mathrm{MeV} / \mathrm{c}, R$ en $\mu \mathrm{m}$ et $M$ en U.M.A., le lissage des courbes permet d'obtenir, avec $N=M / 10$ :

- pour $R \lesssim 7 \mu \mathrm{m}$ :

$$
\begin{aligned}
& A=5,6782+29,9065 N-2,0506 N^{2} \\
& +0,1900 N^{3}-0,0080 N^{4} \\
& B=57,3160-44,1664 N+62,8528 N^{2} \\
& -11,3786 N^{3}+0,5624 N^{4} \\
& \text { - pour } 7 \mu \mathrm{m}<R \lesssim 25 \mu \text { : } \\
& A=-21,0569+45,3713 N-9,6023 N^{2} \\
& +1,2373 N^{3}-0,0494 N^{4} \\
& B=175,1736-31,2438 N+62,9793 N^{2} \\
& -10,8247 N^{3}+0,4767 N^{4} \text {. }
\end{aligned}
$$

Dans le cas où la masse de l'ion est supérieure à 90 et inférieure à 140 , on a, en première approximation :

$$
\begin{aligned}
& A=(0,97 M+110,37) \\
& B=(-4,12 M+651,4)
\end{aligned}
$$

pour des parcours compris entre 1 et $40 \mu \mathrm{m}$.

Les expressions analytiques ainsi déterminées nous sont très utiles dans l'étude des différentes réactions nucléaires afférentes à la fission et à la spallation à haute énergie. Ces considérations nous permettront en particulier d'obtenir la distribution des masses dans la fission ternaire, dont l'étude peut difficilement s'effectuer à l'aide de compteurs ou de jonctions.

III. Application. - Les courbes parcours-énergie et parcours-quantité de mouvement nous ont permis, en première application, de déterminer les distribu- 
tions des masses, des charges et des énergies cinétiques dans la fission de l'uranium par neutrons thermiques. Les résultats qui sont parfaitement connus nous permettent de vérifier le bien-fondé de notre étude sur un exemple pratique.

a) Technique utilisée. - La technique utilisée est celle du « sandwich » [21], [23]. Rappelons brièvement la méthode.

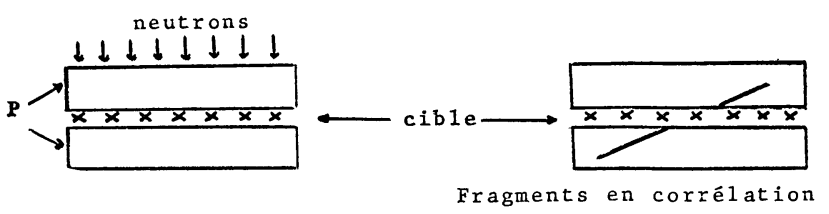

Sur une partie de la surface d'une feuille de plastique (makrofol ou nitrate de cellulose), nous déposons par évaporation une couche de $\mathrm{U}_{3} \mathrm{O}_{8}\left(\right.$ ou $\left.\mathrm{UF}_{4}\right)$ de quelques centaines d'Å. Cette couche constituera la cible de U. Par-dessus et contre la couche ainsi évaporée, sera appliquée une feuille de plastique semblable à la première. Les deux feuilles, suffisamment épaisses pour avoir de la tenue $(\simeq 300 \mu)$, seront fortement collées ensemble sur toutes leurs parties extérieures à la couche de $\mathrm{U}_{3} \mathrm{O}_{8}$ (ou $\mathrm{UF}_{4}$ ), en prenant soin toutefois de laisser quelques petites ouvertures permettant la dissolution de la cible et, ultérieurement, le développement chimique des traces. L'ensemble détecteurcible sera en général irradié perpendiculairement à sa surface.

Le dispositif ainsi conçu permet de visualiser des phénomènes en corrélation provenant de la fission d'un même noyau d'uranium.

Les fragments de fission seront visibles chacun dans une des deux feuilles accolées. L'observation se fera au microscope optique. On mesurera la longueur et l'enfoncement de chaque fragment, ainsi que l'angle des deux fragments en corrélation. L'angle entre les deux fragments est égal à $180^{\circ}$ dans le cas de la fission de l'uranium par neutrons thermiques.

Pour pouvoir observer des phénomènes en corrélation avec un maximum d'efficacité, il faut que :

- Pendant l'exposition et pendant l'observation au microscope, les deux feuilles de plastique restent intimement plaquées l'une contre l'autre à l'échelle microscopique;

- Durant le développement chimique, l'ensemble des deux feuilles n'ait subi aucun glissement, l'une par rapport à l'autre.

Le fait d'avoir collé les bords sur une grande étendue empêche en principe tout glissement. Pour plus de sûreté, durant l'exposition et l'observation, le détecteur est préalablement mis sous vide dans un sachet de plastique.

Nous avons étudié 1450 fragments de fission en corrélation deux à deux. A partir des mesures des parcours visibles dans le détecteur plastique, nous avons déduit les courbes de distribution des masses, des charges et d'énergie.

b) Distribution des masses, Des Gharges et Des ÉNERGIES GINÉTIQUES DANS LA FISSION DE L'URANIUM PAR NEUTRONS THERMiQUES. - Pour exécuter le calcul, nos hypothèses de travail ont été les suivantes :

1) Conservation de la quantité de mouvement $\mathbf{P}_{\mathbf{1}}+\mathbf{P}_{\mathbf{2}}=\mathbf{0}$.

2) Conservation de la masse du noyau fissionnant, à 4 unités près (on sait qu'en moyenne il y a éjection de 2,5 neutrons) :

$$
232 \leqslant M_{1}+M_{2} \leqslant 236
$$

$M_{1}$ et $M_{2}$ représentent les masses des fragments.

3) Conservation des charges :

$$
Z_{1}+Z_{2}=92
$$

$Z_{1}$ et $Z_{2}$ sont les nombres de charge des fragments.

4) Le rapport $M / Z=2,55$. On sait en effet que pour les fragments légers $M_{1} / Z_{1}=2,552$, et pour les fragments lourds $M_{2} / Z_{2}=2,555$. Le rapport 2,55 est donc a priori convenable.

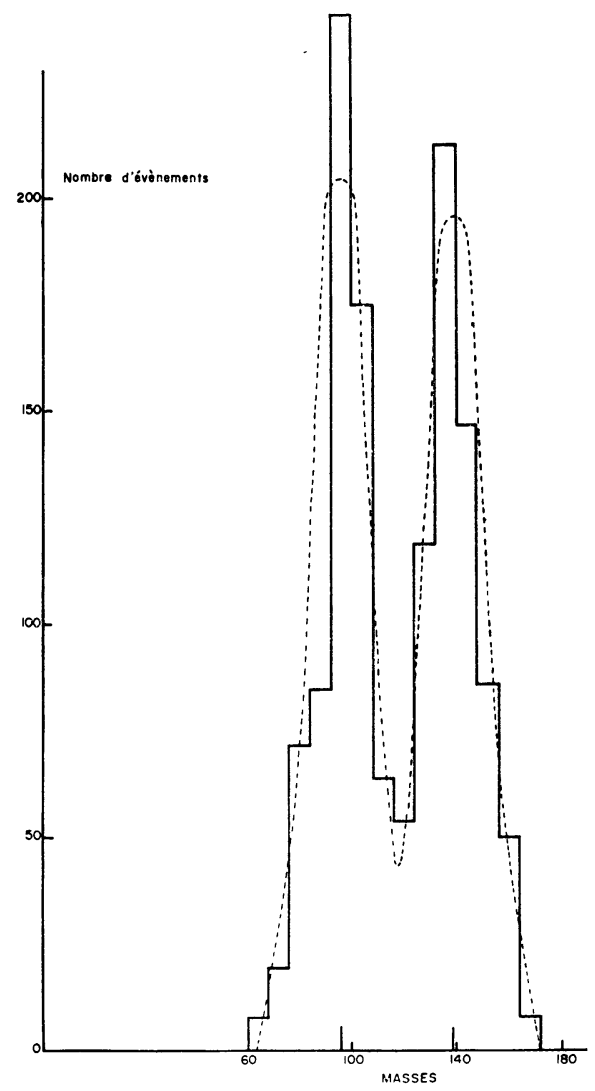

FIG. 10. - Fission de l'uranium par neutrons thermiques. Distribution des masses obtenue par la méthode des détecteurs ionographiques solides. 
Nous avons tracé un réseau de courbes représentant la quantité de mouvement en fonction du parcours ( fig. 8). Partant de ce réseau de courbes et connaissant les parcours $R_{1}$ et $R_{2}$ des deux fragments, nous pouvons déduire les distributions de masses, de charges et d'énergies dans la fission de l'uranium par neutrons thermiques. On obtient les valeurs suivantes :

Fragments légers :

Charge moyenne ........ 38

Masse moyenne ......... 96

Énergie la plus probable ... $95 \mathrm{MeV}$

Fragments lourds :

Charge moyenne ........ 54

Masse moyenne .......... 139

Énergie la plus probable... $65 \mathrm{MeV}$

Ge procédé utilisé ici à titre de vérification est destiné à être appliqué dans l'avenir aux interactions dont on voudra étudier les distributions en masse, charge et énergie.

IV. Examen et discussion du procédé de calcul utilisé. - a) RÉsultats OBTENUS PAR D'AUTRES AUTEURS. - Dans de très récentes publications, différents auteurs ont étudié les relations parcours-énergie et les

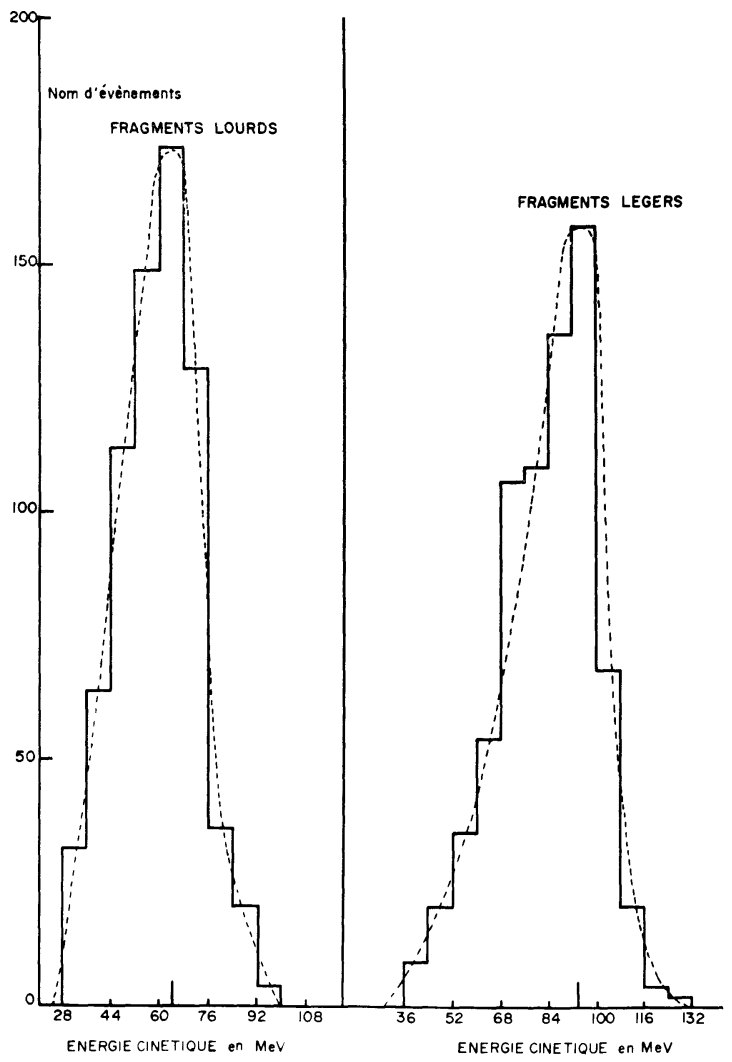

FIG. 11. - Fission de l'uranium par neutrons thermiques. Distribution de l'énergie cinétique obtenue par la méthode des détecteurs ionographiques solides.

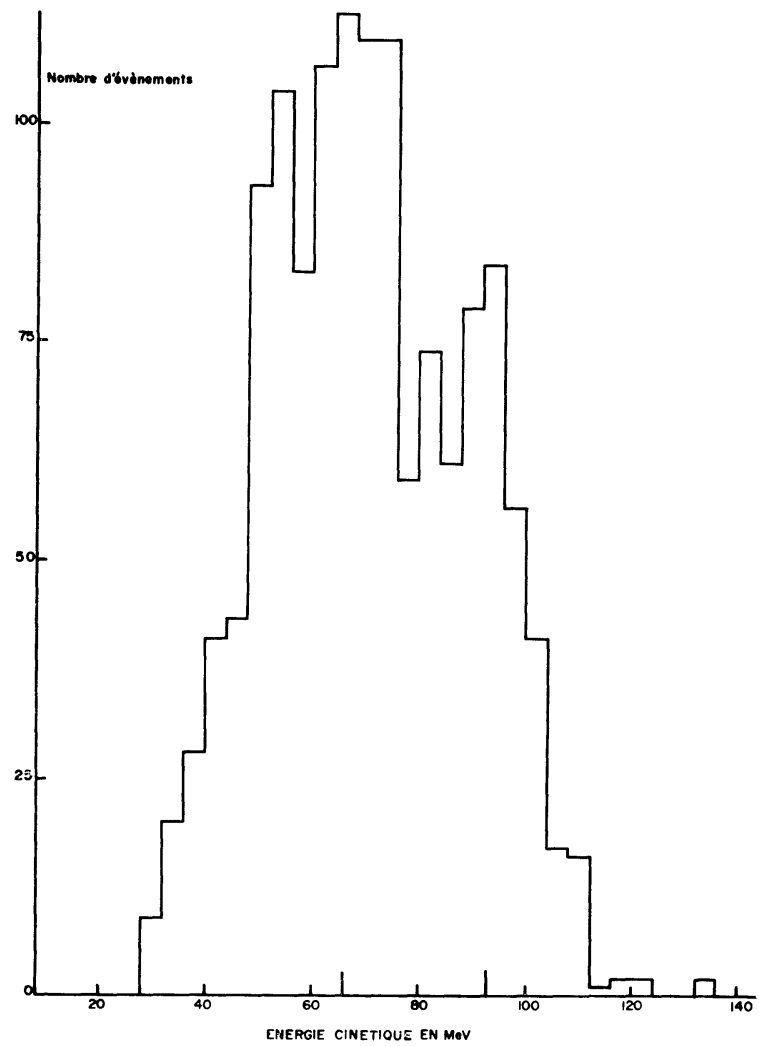

FIG. 12. - Fission de l'uranium par neutrons thermiques. Distribution de l'énergie cinétique obtenue par la méthode des détecteurs ionographiques solides.

pertes spécifiques d'énergie dans certains détecteurs ionographiques visuels solides. Passons très rapidement en revue les principaux résultats qui ont été obtenus jusqu'à présent.

Benton et Henke [24] ont fait une étude très étendue des relations parcours-énergie et perte spécifique d'énergie dans différents matériaux plastiques, et en particulier dans le nitrate de cellulose. Leur méthode, basée sur un calcul théorique utilisant la relation d'Heckmann et Barkas d'une part et celle de Barkas et Berger d'autre part, leur a permis de déterminer le parcours et la perte spécifique d'énergie d'un ion, de masse comprise entre 1 et 84 . Leur gamme d'énergie est très large, allant de 0,10 à $1200 \mathrm{MeV}$ /nucléon.

Ait-Salem et coll. (Physikalisches Institut der Universität Tübingen) [25] ont étudié les distributions de masse et d'énergie des fragments de fission à partir de leur parcours dans le nitrate de cellulose. Ils ont défini une relation de la forme $R \propto E^{a} A^{b}$ (où $E$ est l'énergie et $A$ le nombre de masse), dans laquelle ils ont déterminé expérimentalement les constantes $a$ et $b$.

Cumming et Crespo (Brookhaven National Laboratory) [26] ont mesuré, pour quelques fragments de fission, les pertes d'énergie et les parcours dans le mylar. Les résultats qu'ils ont obtenus sont conformes à la 
théorie de la perte d'énergie par chocs électroniques, dans laquelle est introduite une expression de la « charge fractionnaire efficace », fonction universelle de la variable $v / v_{0} Z^{2 / 3}$, où $v$ est la vitesse de l'ion et $Z$ son nombre de charge.

b) CAS DU MAKROFOL. - Nous nous sommes penchés spécialement sur le cas du makrofol pour une raison de commodité expérimentale, et du fait qu'à notre avis ce détecteur se prête particulièrement bien à l'étude de la fission ternaire. La méthode de calcul reste la même pour le nitrate de cellulose ainsi que pour d'autres détecteurs visuels plastiques. L'ajustement de certains coefficients intervenant dans la détermination des parcours a été rendu possible grâce à des expériences effectuées à l'accélérateur d'ions lourds de Heidelberg (Allemagne). Rappelons que nous nous intéressons en priorité aux ions lourds de faible vitesse et principalement aux ions de fission.

Les parcours que nous donnons ne sont pas nécessairement les parcours enregistrés dans le makrofol. Si la perte spécifique d'énergie $(\mathrm{d} E / \mathrm{d} R)$ est inférieure à celle d'un ion oxygène de $40 \mathrm{MeV}$, la trace de l'ion n'est pas révélable. En fait, la particule créera des défauts dans le plastique, mais ceux-ci seront expérimentalement peu exploitables et ne peuvent être visualisés par les moyens habituels. Par exemple, les particules $\alpha$ peuvent être détectées dans du makrofol, mais nécessitent un développement à la soude à $20 \%$ et $70^{\circ} \mathrm{C}$ pendant 4 à 6 heures. Les défauts, visibles au microscope optique, apparaissent alors comme des points noirs.

Nous avons tracé la courbe expérimentale de variation du parcours en fonction de l'énergie pour l'ion oxygène ( fig. 13). Cette courbe représente le parcours effectivement enregistré.

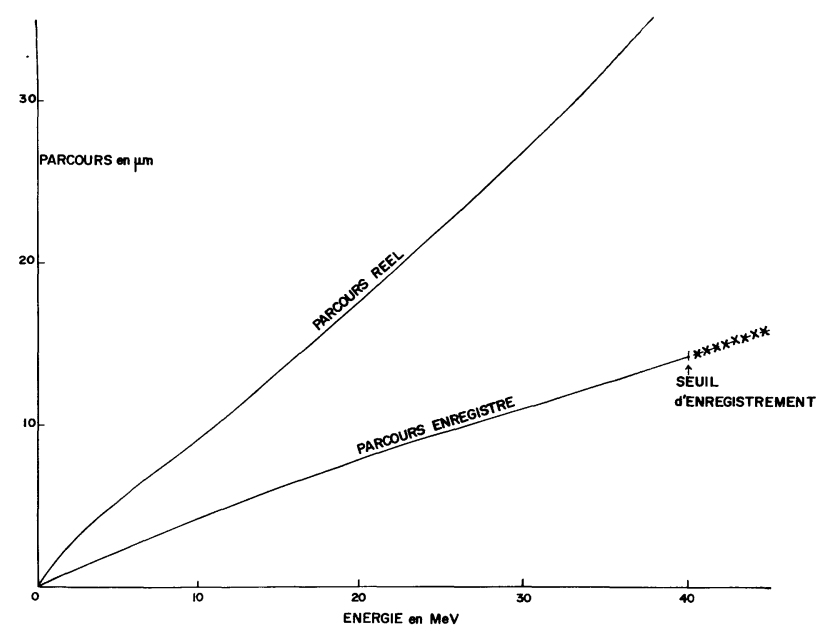

FIG. 13. - Courbes parcours-énergie de l'oxygène 16 dans le makrofol.

En ce qui concerne l'enregistrement des traces, il est à noter que les conditions d'attaque chimique jouent un rôle extrêmement important, en particulier dans le cas des détecteurs sensibles, comme le nitrate de cellulose [15]. Il faut que la vitesse de diffusion de la soude dans le canal constituant la trace soit supérieure à celle du rongement de la surface du détecteur par l'agent chimique de développement. D'où la nécessité d'un dosage adéquat des facteurs tempsconcentration-température suivant la perte spécifique d'énergie, la longueur et l'enfoncement de la trace.

En observant les courbes $E=f(R)$ des figures 4 à 6 , on remarque l'existence de points d'inflexion aux abscisses suivantes :

$\begin{array}{ccccccc}Z & 8 & 11 & 13 & 14 & 15 & 20 \\ E(\mathrm{MeV}) & 6,4 & 13,75 & 20,25 & 24,30 & 28,80 & 58,60\end{array}$

Ces points d'inflexion correspondent aux maxima d'ionisation dus aux chocs électroniques. Ces maxima se produisent pour les vitesses $v_{1}$ des ions telles que $v_{1}=v_{0} Z^{2 / 3}\left(v_{0}:\right.$ vitesse de Bohr) [9].

c) Autre possibilité de Galgul dans le GAS DES FRAGMENTS DE FISSION. - N. Bohr [27] avait remarqué qu'il existe une analogie entre le parcours d'une particule $\alpha$ et celui d'un fragment de fission de même vitesse. Il a donné la relation suivante, reliant le parcours $R_{\alpha}$ des particules $\alpha$ au parcours $R_{\mathrm{F}}$ des ions de fission :

$$
\frac{R_{\mathrm{F}}}{R_{\alpha}}=7 \frac{A}{\left(Z_{\text {eff }}\right)^{2}}=7 \frac{A}{Z^{2 / 3}}\left(\frac{e^{2}}{h v}\right)^{2}
$$

Segre et Wiegand [28] avaient étudié comparativement les pouvoirs d'arrêt de $\mathrm{Al}, \mathrm{Cu}, \mathrm{Ag}, \mathrm{Au}$ et collodion (nitrate de cellulose) pour des fragments de fission et pour les particules $\alpha$ de $4,66 \mathrm{MeV}$. La relation de Bohr est en très bon accord avec les résultats expérimentaux de ces auteurs en ce qui concerne les matériaux lourds; elle l'est beaucoup moins dans le cas de la nitrocellulose.

Pour les matériaux constitués d'éléments légers, la divergence des résultats a été prévue et discutée par Bohr lui-même [27].

En opérant sur le makrofol, matériau constitué de noyaux très légers, l'expérience nous a conduits au coefficient numérique 4,3 , différent du coefficient 7 
trouvé par Bohr dans les matériaux plus lourds que l'aluminium.

Nous avons alors dans le makrofol :

$$
\frac{R_{\mathrm{F}}}{R_{\alpha}}=4,3 \frac{A}{\left(Z_{\text {eff }}\right)^{2}}=4,3 \frac{A}{Z^{2 / 3}}\left(\frac{e^{2}}{h v}\right)^{2} .
$$

De cette relation, nous tirons :

$$
R_{\mathrm{F}}=0,23\left(\frac{A}{Z}\right) Z^{1 / 3} 10^{-3} \frac{R_{\alpha}}{\beta^{2}} .
$$

Posons $\frac{A}{Z}=2,55$ et $10^{-3} R_{\alpha} / \beta^{2}=K(\beta)$.

Nous avons alors :

$$
R_{\mathrm{F}}=0,59 Z^{1 / 3} K(\beta)
$$

$K(\beta)$ est une fonction de $\beta$, caractéristique du matériau considéré. Nous avons tracé la courbe $K(\beta)=$ fonction de $\beta$ ( fig. 14).

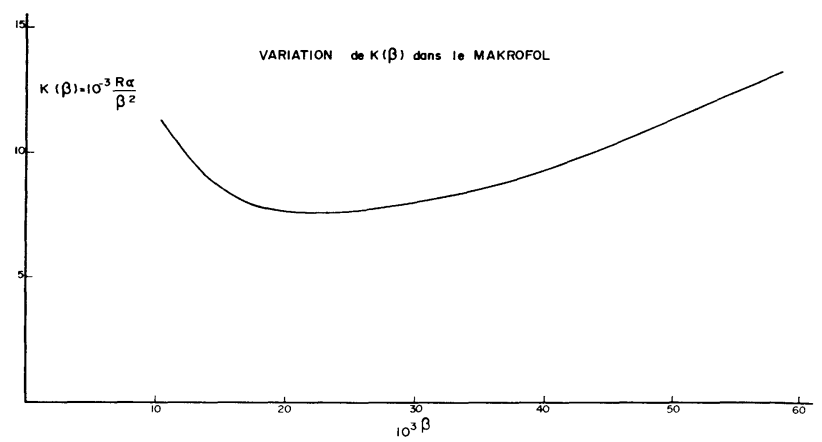

FIG. 14. - Variation de $K(\beta)$ dans le makrofol.

Conclusion générale. - Comme nous l'avons déjà indiqué, nous nous proposons, dans un proche avenir, d'utiliser cette méthode de calcul dans l'étude cinétique, et en particulier de l'appliquer à la détermination des masses dans le cas de la fission ternaire. Le premier problème consiste à obtenir, pour les frag- ments de fission et pour les ions plus légers, c'est-à-dire dans un domaine très large de masses et de parcours, une ou plusieurs relations analytiques du type $P=f(R, M)$ nous permettant de relier la quantité de mouvement $P$, la masse $M$ et le parcours $R$ d'un fragment lourd quelconque, avec une bonne précision. Comme nous l'avons montré, cette phase est déjà largement entamée et il ne nous reste à l'heure actuelle qu'à étudier, expérience à l'appui, la zone de limite d'enregistrement, c'est-à-dire la zone des masses comprises entre 16 et 20 , et cela pour des parcours compris entre 1 et $40 \mu \mathrm{m}$. Nous serions alors à même de terminer et d'exploiter notre programme de calcul des événements ternaires. Nous avons, à l'heure actuelle, commencé l'étude de la fission ternaire dans l'uranium à haute énergie. Dans ce cas, on ne trouve que très rarement des fragments de masse inférieure à 20; alors que l'étude de la fission dans le cas du Bi et du $\mathrm{Pb}$ nous conduira, comme le montrent nos résultats préliminaires, à des masses assez faibles. Nous pensons, d'ici quelques mois, être à même de donner des distributions en masses, au moins dans le cas de l'uranium et du plomb.

Ce travail a été exécuté au Laboratoire de Physique Corpusculaire de Strasbourg, dirigé par M. le Professeur P. Cüer.

Remerciements. - Nous tenons spécialement à remercier l'équipe de l'accélérateur de Heidelberg en Allemagne qui nous a permis les expositions aux ions de différentes masses et énergies. Nous remercions en particulier le Docteur Bock pour le temps qu'il a bien voulu nous consacrer et pour les facilités qu'il nous a ménagées autour de l'accélérateur.

Nous remercions également MM. C. Muller et A. Kappler, Mme Cartier, ainsi que Mlles M. Munch, A. M. Dester, M. J. Bossard, M. A. Alegre, B. Giess, faisant partie de l'équipe de techniciens du Laboratoire, qui nous ont permis la mise en œuvre des cibles et les mesures souvent fastidieuses des événements.

\section{BIBLIOGRAPHIE}

[1] Sirk (E. Ch.) et BARnES (R. S.), Phil. Mag., 1956, 4, 970 .

[2] Price (P. B.) et Walker (R. M.), Phys. Rev. Letters, 1962, 8, 217.

[3] Debeauvais (M.), Libourel (F.) et Mulíer (C.), Réunion de travail sur l'enregistrement des traces de particules chargées dans les cristaux, Strasbourg, 13-14 mai 1963.

[4] Debeauvais (M.) et CüER (P.), Réunion de travail de Strasbourg, 1963 (déjà citée).

[5] WALKER (R. M.), Réunion de travail de Strasbourg, 1963 (déjà citée).

[6] Debeauvais (M.) et Monnin (M.), C. R. Acad. Sci. Pavis, 1965, 260, 4728.

[7] Fleischer (R. L.), Price (P. B.), WALkeR (R. M.) et Hubbard (E. L.), Phys. Rev., 1964, 133, A 1443.
[8] Stein (R.), Mosinsky (G.) et Ralarosy (J.), Dosage de rayonnement et de flux de grande énergie au moyen d'événements enregistrés dans les hauts polymères, Colloque Internat. sur "L’Électronique Nucléaire et la Radioprotection ", Toulouse, 5-8 mars 1968.

[9] LinhaRd (J.), SchaRfF (M.) et SchioTT (H. E.), Mat. Fys. Medd. Dan. Vid. Selsk., 1963, 33, 14.

[10] CüER (P.), Réunion de travail de Strasbourg, 1963 (déjà citée).

[11] Fleischer (R. L.), Price (P. B.), WalkeR (R. M.) et Hubbard (E. L.), Phys. Rev., 1967, 156, 353.

[12] Fleischer (R. L.), Price (P. B.) et WalkeR (R. M.), J. Appl. Phys., 1965, 36, 3645.

[13] Pfohl (R.), Monnin (M.) et Debeauvais (M.), C. R. Acad. Sci. Paris, 1965, 261, 2216.

[14] Benton (E. V.), USNR, TR 67, July 30, 1967. 
[15] Tripier (J.), Debeauvais (M.), Stein (R.) et Ralarosy (J.), Contribution à l'étude méthodologique de l'enregistrement d'ions lourds dans le nitrate de cellulose, J. Micros., 1968, 7, 6.

[16] BETHE (H. A.) et Ashkin (J.), Experimental Nuclear Physic, vol. I, Segre Editor.

[17] Evans (R. D.), The Atomic Nucleus, McGraw-Hill Book Company, 1955.

[18] Barkas (W. H.), Nuclear Research Emulsions, Academic Press, 1963.

[19] Heckmann (H. H.), Perkins (B. L.), Simon (W. G.), Smith (F. M.) et BARkas (W. H.), Phys. Rev., 1960, 117.

[20] Vigneron (L.), C. R. Acad.Sc. Paris, 1950, 231, 1473.

[21] Debeauvais (M.), Stein (R.), RÉmy (G.) et RaLARosy (J.), C. R. Acad. Sc. Paris, 1966, 263, 398.

[22] Debeauvais (M.), Stein (R.), Rémy (G.) et RalaRosy (J.), Suppl. J. Physique, 1, 1968, 29, C1-127.
[23] Debeauvais (M.), Stein (R.), Ralarosy (J.) et CÜER (P.), Nuclear Phys., 1967, A 90, 186.

[24] Benton (E. V.) et Henke (R. P.), USNRDL TR 1102, nov. 1966.

[25] Ait-Salem (M.), GerhardT (H.), GönNenwein (F.), HIPP et PAAP (H.), Nucl. Inst. and Meth., 1968, 60, 1.

[26] Cumming (J. B.) et Crespo (V. P.), Energy loss and range of fission fragments in solid media, à paraître.

[27] BoHr (N.), Kgl. Danske Videnskab. Selskab. Mat. Fys. Medd., 1948, 18, 8.

[28] Segre (E.) et Wiegand (C.), Phys. Rev., 1946, 70, 808.

[29] Demers (R.), Les émulsions nucléaires, Presses Universitaires de Montréal. 